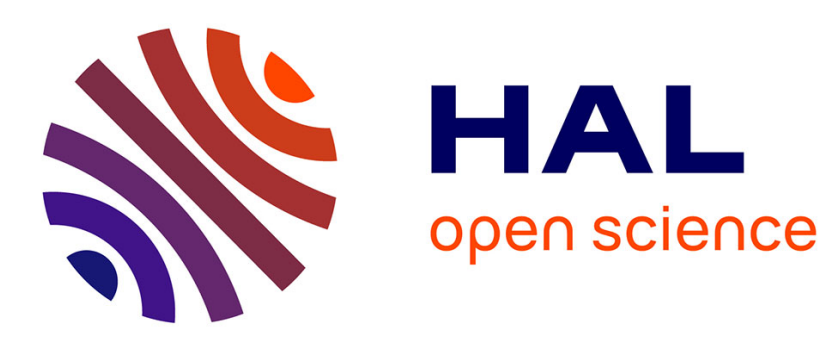

\title{
A Central Limit Theorem for the Generalized Quadratic Variation of the Step Fractional Brownian Motion
}

Antoine Ayache, Pierre Bertrand, Jacques Lévy Véhel

\section{To cite this version:}

Antoine Ayache, Pierre Bertrand, Jacques Lévy Véhel. A Central Limit Theorem for the Generalized Quadratic Variation of the Step Fractional Brownian Motion. Statistical Inference for Stochastic Processes, 2007, 10 (1), pp.1-27. 10.1007/s11203-005-0532-2 . hal-00539211

\section{HAL Id: hal-00539211 \\ https://hal.science/hal-00539211}

Submitted on 24 Nov 2010

HAL is a multi-disciplinary open access archive for the deposit and dissemination of scientific research documents, whether they are published or not. The documents may come from teaching and research institutions in France or abroad, or from public or private research centers.
L'archive ouverte pluridisciplinaire HAL, est destinée au dépôt et à la diffusion de documents scientifiques de niveau recherche, publiés ou non, émanant des établissements d'enseignement et de recherche français ou étrangers, des laboratoires publics ou privés. 


\section{A central limit theorem for the generalized quadratic variation of the step fractional Brownian motion}

Antoine Ayache

Laboratoire Paul Painlevé, Bât. M2,

Université Lille 1, 59655 Villeneuve d'Ascq, France.

And

IAE de Lille, 104, Avenue du Peuple Belge 59043 Lille Cedex, France.

E-mail : Antoine.Ayache@math.univ-lille1.fr

Pierre Bertrand

Laboratoire de Mathématiques (UMR CNRS 6620)

Université Blaise Pascal (Clermont-Ferrand II), 63177 Aubière Cedex, France.

E-mail : bertrand@math.univ-bpclermont.fr

Jacques Lévy Vehel

INRIA Rocquencourt

Domaine de Voluceau BP 105

78153 Le Chesnay Cedex, France.

E-mail : Jacques.Levy-Vehel@inria.fr

Abstract : This paper gives a central limit theorem for the generalized quadratic variation

of the step fractional Brownian motion. We first recall the definition of this process and the statistical results on the estimation of its parameters.

Key Words: Step fractional Brownian motion, Hurst index, detection of abrupt changes, random wavelet series, generalized quadratic variation.

Abbreviations : FBM : Fractional Brownian Motion, MBM : Multifractional Brownian Motion, SFBM : Step Fractional Brownian Motion; r.v. : random variable. 


\section{Introduction}

Fractional Brownian Motion (FBM) was introduced in 1940 by Kolmogorov as a way to generate Gaussian "spirals" in a Hilbert space. But the seminal paper of Mandelbrot and Van Ness (1968) emphasizes the relevance of FBM to the modelling of natural phenomena : Hydrology, Finance,... Various properties of FBM such as long range dependence, self-similarity and smoothness of the sample paths are governed by the Hurst parameter $H$. During the decades 1970's and 1980's, the statistical study of FBM has been developed, see for instance the historical notes in Samorodnitsky \& Taqqu (1994), [16, chap.14] and the references therein. FBM has been more and more used in several areas during the last decade (internet traffic, turbulence, image processing...). Anyway, in many applications the real data do not fit exactly FBM which appears as an ideal mathematical model : for example, a constant Hurst parameter is a too rigid assumption in many applications, such as in finance or in turbulence, see Papanicolaou and Sølna (2002). Therefore different generalizations of FBM have been proposed these last years to fill the gap between the mathematical modelling and real data. One example is the family of model derived from the Multifractional Brownian Motion (MBM) introduced independently in Peltier and Lévy Vehel (1996) and Benassi et al. (1997). For the MBM the Hurst parameter $H$ is replaced by a function depending continuously on the time $t \mapsto H(t)$. Finding a good generalization of the FBM enhancing the goodness of fit to the different applications has become a fashionable sport, played by several authors, see for instance the work of Cheridito (2003) motivated by financial theory and empirical econometric evidences; Bardet and Bertrand (2003) motivated by applications in biomechanics or Ayache and Lévy Véhel (2000) motivated by applications in signal processing and turbulence .

The MBM was defined for a Hurst parameter being a function depending continuously on time $H($.$) , but in some fields (image analysis or control of internet traffic) the interesting information$ is the location of the change points of the function $H($.$) . The Step Fractional Brownian Motion$ (SFBM) which was introduced in [5], is defined as a generalization of the FBM with a piecewise constant function $H($.$) . It shares some nice properties of FBM, see [5]. A statistical study of$ the SFBM has already been performed in [5], namely an almost surely convergent estimator of its parameters, based on the generalized quadratic variation, has been introduced.

At this stage, we should insist on the following point. In the literature, the generalized quadratic variation is often used for estimating a Hurst type index because, contrarily to the usual quadratic variation, it follows a standard Central Limit Theorem, see for instance Guyon and Leon (1989), Istas and Lang (1997) or Bardet (2000). This result is explained more precisely in section 3 below. Let us mention that in a rather different setting Davydov and al. (2003) propose a statistical index for measuring the fluctuations of a stochastic process $X$. This index is based on generalization of Lorenz curves and Gini index. Davydov and al. develop a complete 
picture of the asymptotic behaviour and show that the fluctuation indices goes through to a phase transition at $H=3 / 4$ and they are the first to provide results in the critical case $H=3 / 4$.

The almost sure convergence of the estimator of the parameters of the SFBM is a first step of the statistical study. In applications, we would like to test the existence of one change point, estimate the probability of error of type 1 or 2 and have confidence intervals for the estimated parameters. In both case, we need the knowledge, at least asymptotically, of the law of the estimator and since the estimator is derived from the generalized quadratic variation, we need a Central Limit Theorem for the generalized quadratic variation.

In this work, we prove a Central Limit Theorem for the generalized quadratic variations of SFBM. The remainder of the paper is organized as follows; in section 2, we recall the definition of the SFBM and the statistical problem: this process is defined as a wavelet series which is a generalization of the wavelet expansion of the FBM. In section 3, we justify the use of generalized quadratic variation instead of the usual one: the generalized quadratic variation follows a standard Central Limit Theorem without any phase transition phenomenon. Then we state our main result which consists in a standard Central Limit Theorem for the generalized quadratic variation of SFBM. This result is proved in Section 4 and rely on a deterministic Lemma related to wavelets given in Appendix A and probabilistic Lemmas established in Appendix B.

\section{Description of the problem}

\subsection{Recall on the FBM and its wavelet series expansion}

A fractional Brownian motion $B_{H}=\left\{B_{H}(t), t \in \mathbb{R}\right\}$ of parameters $\left.H \in\right] 0,1[$ and $\sigma>0$ is a real valued centered Gaussian process with stationary increments that satisfies $B_{H}(0)=0$ and $\mathbb{E}\left|B_{H}(s)-B_{H}(t)\right|^{2}=\sigma^{2}|t-s|^{2 H}$ for every reals $s, t$. The Hurst parameter $H$ governs different properties of the FBM : for instance, the rate of correlation of the increments of $B_{H}$ (long range dependence) ; the self-similarity of the process, i.e. for all $\lambda \in \mathbb{R}_{+}$,

$$
\left(B_{H}(\lambda t)\right)_{t \in \mathbb{R}_{+}} \stackrel{(d)}{=}\left(\lambda^{H} B_{H}(t)\right)_{t \in \mathbb{R}}
$$

the Hölder regularity of the paths. FBM has several representations, see Samorodnitsky \& Taqqu (1994), [16, chap. 7]. Here we use its harmonizable representation,

$$
B_{H}(t)=\int_{\mathbb{R}} \frac{\left(e^{i t \xi}-1\right)}{|\xi|^{H+1 / 2}} \times \widehat{W}(d \xi)
$$

where $W(d x)$ is a Brownian measure and $\widehat{W}(d \xi)$ its Fourier transform, namely for any function $f \in L^{2}(\mathbb{R})$ one has almost surely, $\int_{\mathbb{R}} f(x) W(d x)=\int_{\mathbb{R}} \widehat{f}(\xi) \widehat{W}(d \xi)$, with the convention that $\widehat{f}(\xi)=\int_{\mathbb{R}} e^{-i \xi x} f(x) d x$ when $f \in L^{1}(\mathbb{R}) \cap L^{2}(\mathbb{R})$. From the harmonizable representation (1), 
we derive the wavelet expansion of the FBM. Let us recall the definition of a Lemarié-Meyer wavelet basis of $L^{2}(\mathbb{R})$.

1. The functions $\psi_{j, k}$ defined as $\psi_{j, k}(x)=2^{j / 2} \psi\left(2^{j} x-k\right)$ are generated by dilations and translations of a unique function $\psi$ called a "mother wavelet". Observe that $\psi=\psi_{0,0}$.

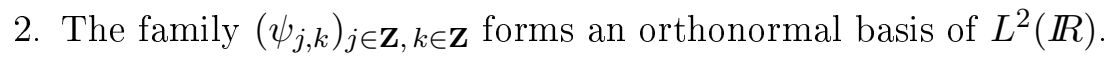

3. The functions $\psi_{j, k}$, and then $\widehat{\psi_{j, k}}$, belong to the Schwartz class $\mathcal{S}(\mathbb{R})$, where $\mathcal{S}(\mathbb{R})$ is the space of all infinitely differentiable functions $f$ whose derivatives $f^{(n)}$ of any order $n \geq 0$ satisfy for all integer $m, \lim _{|t| \rightarrow \infty} t^{m} f^{(n)}(t)=0$.

Moreover the support of $\widehat{\psi}$ is contained in the compact domain $\{\xi \in \mathbb{R}, 2 \pi / 3 \leq|\xi| \leq 8 \pi / 3\}$. We denote $\varphi$ a corresponding scaling function and for every $l \in \mathbf{Z}$ we denote $\varphi_{l}$ the function $x \mapsto \varphi(x-l)$. We will mainly use the following properties : $\widehat{\varphi}$ has a compact support, $\varphi$ and $\widehat{\varphi}$ belong to the Schwartz class $\mathcal{S}(\mathbb{R})$ and the family $\left\{\varphi_{l}, \psi_{j, k}, j \in \mathbb{N}, k \in \mathbf{Z}, l \in \mathbf{Z}\right\}$ forms an orthonormal basis of $L^{2}(\mathbb{R})$. The decomposition of the kernel $\xi \mapsto \frac{\left(e^{i t \xi}-1\right)}{|\xi|^{H+1 / 2}}$ of the representation (1) in the basis $\left\{\widehat{\varphi}_{l}, \widehat{\psi}_{j, k}, j \in \mathbb{N}, k \in \mathbf{Z}, l \in \mathbf{Z}\right\}$ provides the following expansion of the FBM

$$
B_{H}(t)=\sum_{j \in \mathbb{N}, k \in \mathbf{Z}} \Phi_{j, k}(t, H) \zeta_{j, k}+\widetilde{B}_{H}(t)
$$

with

$$
\widetilde{B}_{H}(t)=\sum_{l \in \mathbf{Z}}\left\{\int_{\mathbb{R}} \frac{\left(e^{i t \xi}-1\right)}{|\xi|^{H+1 / 2}} e^{-i l \xi} \overline{\hat{\varphi}_{l}(\xi)} d \xi\right\} \eta_{l}
$$

where

- $\left\{\eta_{l}, \zeta_{j, k} ; j \in \mathbb{N}\right.$ and $\left.k, l \in \mathbf{Z}\right\}$ is a sequence of independent $\mathcal{N}(0,1)$ Gaussian r. v.

- the functions $\Phi_{j, k}(t, y)$ are defined by

$$
\Phi_{j, k}(t, y)=\int_{\mathbb{R}} \frac{\left(e^{i t \xi}-1\right)}{|\xi|^{y+1 / 2}} \widehat{\widehat{\psi_{j, k}}}(\xi) d \xi
$$

The terms $\widetilde{B}_{H}(t)$ and $\sum_{j \in \mathbb{N}, k \in \mathbf{Z}} \Phi_{j, k}(t, H) \zeta_{j, k}$ respectively corresponds to the "low frequencies" and the "high frequencies" components of the wavelet expansion (2) of the FBM. 


\subsection{Definition of the Step fractional Brownian motion.}

The Step Fractional Brownian Motion (SFBM) is an extension of FBM in which the Hurst parameter $H$ has been replaced by a piecewise constant function $H($.$) . More precisely, H($.$) is$ with values in $(0,1)$ and can be written for every real number $t$ as

$$
H(t)=\Sigma_{i=0}^{K} a_{i} \mathbf{1}_{\left[\tau_{i}, \tau_{i+1}[\right.}(t)
$$

where $\tau_{0}=-\infty, \tau_{K+1}=\infty$ and $\tau_{1}, \ldots, \tau_{K}$ is an increasing finite sequence of (non dyadic) real numbers.

A SFBM is defined like a fractional Brownian motion, where we would have replace the constant Hurst index $H$ by the function $t \mapsto H(t)$ in the random wavelet series representation. More formally the SFBM is defined by the random series

$$
X(t)=\sum_{j \in N, k \in \mathbf{Z}} \Phi_{j, k}\left(t, H\left(k 2^{-j}\right)\right) \zeta_{j, k}
$$

where

- $\zeta_{j, k}, j \in \mathbb{N}, k \in \mathbf{Z}$ is a family of standard centred Gaussian random variables.

- The functions $\Phi_{j, k}(t, y)$ are still defined by (4) associated to the mother wavelet $\psi$.

- $H($.$) is the piecewise function given by (5).$

The integer $K$ corresponds to the number of change points of the function $H($.$) . In the case$ $K=0, H($.$) is constant with value a_{0}$ and (6) corresponds to the "high frequencies" component of the wavelet expansion (2) of a FBM with Hurst index $a_{0}$.

\section{A useful representation}

A straightforward calculation, see [5, Formula (5), p.107], shows the equivalence between (6) and the following representation

$$
X(t)=\sum_{j \in I N, k \in \mathbf{Z}}\left\{2^{-j H\left(k / 2^{j}\right)}\left[\Psi_{H\left(k / 2^{j}\right)}\left(2^{j} t-k\right)-\Psi_{H\left(k / 2^{j}\right)}(-k)\right]\right\} \zeta_{j, k} .
$$

where

$$
\Psi_{y}(t)=\int_{\mathbb{R}} e^{i t \xi} \times \overline{\widehat{\psi}}(\xi) \times|\xi|^{-(y+1 / 2)} d \xi
$$

Observe that, as $\widehat{\psi}$ is compactly supported and vanishes in a neighborhood of the origin, the function $\xi \mapsto \frac{\overline{\hat{\psi}}(\xi)}{|\xi|^{y+1 / 2}}$ belongs to the class $\mathcal{S}(\mathbb{R})$. Thus $\psi_{y}$ also belongs to the class $\mathcal{S}(\mathbb{R})$. 


\subsection{The statistical problem}

Let $X$ be a SFBM. We observe one path of the process $X$ at the discrete times $t_{i}=i / N$ for $i \in \mathbf{Z}$. The mesh goes to 0 as $N$ tends to infinity. In [5], the second author has proposed an estimator of the change times for abrupt change greater than a threshold $\eta>0$, i.e. $\left|\delta_{i}\right|>\eta$ where $\delta_{i}=a_{i+1}-a_{i}$. This estimator is based upon the difference between the estimate on a right box and the corresponding one on a left box, both of size $\gamma$ where $\gamma$ is a fixed positive real number. Thus two successive change points, say $\tau_{i}$ and $\tau_{i+1}$, could be detected separately as soon as $\tau_{i+1}-\tau_{i}>2 \gamma$. For this reason and to avoid heavy notations, from now on we restrict to the case of one change point. Under this assumption, the function $H($.$) becomes of the form$

$$
H(t)=a_{0} \mathbf{1}_{\left(-\infty, \tau_{1}\right)}(t)+a_{1} \mathbf{1}_{\left[\tau_{1}, \infty\right)}(t) .
$$

with $a_{0}, a_{1} \in(0,1)$ and $\tau_{1} \in \mathbb{R}$. The change time $\tau_{1}$ is estimated as the first crossing time of the threshold $\eta$ by the function $D_{N}(t)$, i.e. $\widehat{\tau}_{1}^{(N)}=\frac{1}{N} \min \left\{l \in \mathbf{Z}\right.$, tel que $\left.\left|D_{N}(l / N)\right| \geq \eta\right\}$. This function is defined as,

$$
D_{N}(t)=\frac{1}{2 \ln \left(N^{-1}\right)}\left\{\ln \left(V_{N}^{(\mathbf{2})}(t+\gamma)\right)-\ln \left(V_{N}^{(\mathbf{2})}(t)\right)\right\} .
$$

and

$$
V_{N}^{(\mathbf{2})}(t)=\sum_{l=[N t]+1}^{[N t]+N \gamma}\left\{X\left(\frac{l+1}{N}\right)-2 X\left(\frac{l}{N}\right)+X\left(\frac{l-1}{N}\right)\right\}^{2},
$$

is the 2 -variation of step $1 / N$ of the process $X$ onto the box $[t, t+\gamma)$ for a fixed real number $\gamma>0$ (for every real number $x$, we denote $[x]$ its integer part). In [5, Th.2, p.106], the almost sure convergence of the estimator $\widehat{\tau}_{1}^{(N)}$ is proved. This result is derived from the almost sure convergence of $1 / 2+\ln \left(V_{N}^{(\mathbf{2})}(t)\right) /\left(2 \ln \left(N^{-1}\right)\right)$ to $\inf \{H(u), u \in[t, t+\gamma[\}$.

\section{Statement of the main result and justification of the use of Generalized Quadratic Variations}

First a word about notations. Let $X$ be a centered Gaussian process. Let $d=\left(d_{\ell}\right)_{\ell=0, \ldots, p}$ be a finite sequence of real numbers whose first moment vanishes, that is $\sum_{\ell=0}^{p} d_{\ell}=0$. We call $d$-variation of step $1 / N$ the process

$$
V_{N}^{(d)}(t)=\sum_{k=[N t]+1}^{[N t]+N \gamma}\left|Y_{N}(k)\right|^{2}
$$

where

$$
Y_{N}(k)=\sum_{\ell=0}^{p} d_{\ell} X\left(\frac{k+\ell}{N}\right) .
$$


The simplest example is the usual quadratic variation $V_{N}^{(1)}(t)$ which corresponds to $d=(1-1)$ and $Y_{N}(k)=X((k+1) / N)-X(k / N)$. This usual quadratic variation presents the following drawback. Consider the case where $X$ is a FBM with Hurst index $H$, this is the simplest one. Even in this case, the asymptotic behaviour of the quadratic variation changes following $H<3 / 4$ or not, see Formula (14) below. To avoid this phase transition at $H=3 / 4$, one uses the generalized quadratic variation still defined by $(11,12)$ for a finite sequence of real numbers $d$ whose first two moments vanish, namely one has

$$
\sum_{\ell=0}^{p} d_{\ell}=0 \quad \text { and } \quad \sum_{\ell=0}^{p} \ell d_{\ell}=0 .
$$

A simple example is $d=(1,-2,1)$ corresponding to the quadratic variation $V_{N}^{(2)}(t)$ defined by $(10)$.

\section{A justification for the use of Generalized Quadratic Variation}

In this subsection, we restrict ourself to the case of process $X$ being a FBM with a Hurst index $H$. For every mesh $\Delta_{N}>0$, the variogram satisfy $\mathbb{E}\left(\left|X\left(t+\Delta_{N}\right)-X(t)\right|^{2}\right)=\sigma^{2} \Delta_{N}^{2 H}$. Since the random variables $X\left(t+\Delta_{N}\right)-X(t)$ are centered, the empirical estimator of the variogram is

$$
V_{N}^{(\mathbf{1})}=\sum_{i=1}^{N}\left[X\left(t_{i}+\Delta_{N}\right)-X\left(t_{i}\right)\right]^{2},
$$

which is called the usual quadratic variation. Therefore, one of the most "natural" estimator of the Hurst index $H$ is $\widehat{H}_{N}=\frac{\ln V_{N}^{(\mathbf{1})}}{2 \ln \Delta_{N}}$. However, the usual quadratic variation does not follow a standard Central Limit Theorem, and this is a major drawback.

More precisely, Guyon and Leon (1989) have proved that the random variables $N^{2 H} \times V_{N}^{(\mathbf{1})}$ converge almost surely when $N \rightarrow \infty$, but the speed of convergence and the limit law goes through a phase transition at $H=3 / 4$. Actually, we have the following kind of expansion

$$
\frac{V_{N}^{(\mathbf{1})}}{\mathbb{E} V_{N}^{(\mathbf{1})}}=1+\frac{\varepsilon_{N}}{\sqrt{N}}+\left(\sum_{\ell=0}^{p} \ell d_{\ell}\right) N^{-(2-2 H)} \times \zeta_{N}+\mathcal{O}_{P}\left(N^{-(3-2 H)}\right)
$$

where the random variables $\varepsilon_{N}$ converge in law to a Gaussian distribution and the random variables $\zeta_{N}$ converge to a centred non Gaussian distribution which belongs to the second Wiener chaos. This phase transition phenomenon disappears when we replace the usual quadratic variation by the generalized quadratic variation or equivalently assume that the second moment $\sum_{\ell=0}^{p} \ell d_{\ell}$ vanishs. Therefore the generalized quadratic variation follows a standard Central Limit Theorem, that is converges to a Gaussian limit with the rate of convergence $N^{1 / 2}$. We refer for instance, with slightly different frameworks, to Istas and Lang (1997) or Bardet (2000). 


\section{Statement of the main result}

For every integer $N \geq 1$, we set

$$
\mu_{N}^{(d)}(t)=\mathbb{E}\left(V_{N}^{(d)}(t)\right) \quad \text { and } \quad S_{N}^{(d)}(t)=\sqrt{\operatorname{Var}\left(V_{N}^{(d)}(t)\right)} .
$$

Theorem 3.1 Let $X$ be a SFBM defined by (6) and (9) and $V_{N}^{(d)}(t)$ be its d-variation given by (11) and (12). If the sequence d satisfies the conditions (13), then for every real number $t$, we have $V_{N}^{(d)}(t)=\mu_{N}^{(d)}(t)+S_{N}^{(d)}(t) \times \varepsilon_{N}(t)$ where the random variable $\varepsilon_{N}(t)$ converges in distribution to a $\mathcal{N}(0,1)$ Gaussian variable as $N \rightarrow \infty$ and $\mu_{N}^{(d)}(t), S_{N}^{(d)}(t)$ are given by (15).

The proof of Theorem 3.1 is presented in the following section. It relies on some technical lemmas about wavelet series which are proved in the appendices.

Remark 3.1 The condition of nullity of the second moment of d, see (13), is crucial to prove Lemma B.1 (see Remark B.1), which allows to obtain Lemmas B.6 and B.10.

For the sake of simplicity, we set $S_{N}(t)=S_{N}^{(d)}(t)$ in the sequel.

\section{Proof of the main result}

By definition, the $Y_{N}(k)$ are Gaussian centred random variables. In this Gaussian framework, see for instance [11], the Central Limit Theorem is implied by the following property

$$
\lim _{N \rightarrow \infty} \frac{\lambda_{N}(t)}{S_{N}(t)}=0
$$

with $\lambda_{N}(t)=\max \left\{\mu\right.$ for $\mu$ an eigenvalue of the covariance matrix $\left.\operatorname{cov}\left(Y_{N}(i), Y_{N}(j)\right)_{i, j \in I_{N}(t)}\right\}$ where $I_{N}(t)=\{k \in \mathbf{Z}$, such that $[N t]+1 \leq k \leq[N t]+N \gamma\}$ denotes the set of indices. One often bound $\lambda_{N}(t)$ by the quantity

$$
\beta_{N}(t)=\max _{i \in I_{N}(t)} \sum_{j \in I_{N}(t)}\left|\operatorname{cov}\left(Y_{N}(i), Y_{N}(j)\right)\right|
$$

which is less difficult to handle. Relation (16) therefore results from

$$
\lim _{N \rightarrow \infty} \frac{\beta_{N}(t)}{S_{N}(t)}=0
$$

From now on our goal will be to show that Relation (17) holds. The idea behind our proof is that the increments of the SFBM are almost the same as that of an FBM except on the vicinity of the change time. Thus we split the index set $I_{N}(t)$ into three parts : the left of the change 
time, the vicinity of the change time and the right part. More precisely, for any real number $\delta \in(0,1)$, we define

$$
\begin{aligned}
I_{1}^{\delta}(t, N) & =\left\{k \text { such that }[N t]<k \leq[N t]+N \gamma \text { and } k / N<\tau_{1}-3 N^{-\delta}\right\}, \\
I_{2}^{\delta}(t, N) & =\left\{k \text { such that }[N t]<k \leq[N t]+N \gamma \text { and } \tau_{1}-3 N^{-\delta} \leq k / N \leq \tau_{1}+3 N^{-\delta}\right\}, \\
I_{3}^{\delta}(t, N) & =\left\{k \text { such that }[N t]<k \leq[N t]+N \gamma \text { and } \tau_{1}+3 N^{-\delta}<k / N\right\} .
\end{aligned}
$$

Similarly, we denote

$$
J_{1}^{\delta}(t, N)=\left[\frac{[N t]}{N}, \tau_{1}-3 N^{-\delta}\right] \quad \text { and } \quad J_{3}^{\delta}(t, N)=\left[\tau_{1}+3 N^{-\delta}, \frac{[N t]}{N}+\gamma\right]
$$

the convex closure of the sets $1 / N \times I_{1}^{\delta}(t, N)$ and $1 / N \times I_{3}^{\delta}(t, N)$. Since the random variables $Y_{N}(i)$ are centered, we have for every pair of integers $i, j$

$$
\operatorname{cov}\left(Y_{N}(i), Y_{N}(j)\right)=\mathbb{E}\left(Y_{N}(i) Y_{N}(j)\right)
$$

We denote, for $p, q \in\{1,2,3\}$,

$$
G_{p, q}(N, t)=\max _{i \in I_{p}(t, N)} \sum_{j \in I_{q}(t, N)}\left|\mathbb{E}\left(Y_{N}(i) Y_{N}(j)\right)\right|
$$

and

$$
F(t, N)=\max _{i \in I_{N}(t)} \sum_{j \in I_{2}(t, N)}\left|\mathbb{E}\left(Y_{N}(i) Y_{N}(j)\right)\right|
$$

We have

$$
\begin{aligned}
\beta_{N}(t) & \leq F(t, N)+G_{1,1}(t, N)+G_{3,3}(t, N) \\
& +G_{2,1}(t, N)+G_{3,1}(t, N)+G_{1,3}(t, N)+G_{2,3}(t, N) .
\end{aligned}
$$

From Lemma B.4, we bound $F(t, N)$. From Lemma B.6 , we bound $G_{1,1}(t, N)$ and $G_{3,3}(t, N)$. From Lemma B.8, we bound $G_{1,3}(t, N)$ and $G_{3,1}(t, N)$. From Lemma B.9, we bound $G_{2,1}(t, N)$ and $G_{2,3}(t, N)$. Let us precise the notations, from now on we will denote by $x \wedge y$ the infimum of the two real numbers $x$ and $y$. With this notation, we get

$$
\frac{\beta_{N}(t)}{S_{N}(t)}=\frac{\beta_{N}(t)}{N^{1 / 2-2\left(a_{0} \wedge a_{1}\right)}} \times\left[\frac{S_{N}(t)}{N^{1 / 2-2\left(a_{0} \wedge a_{1}\right)}}\right]^{-1},
$$

Lemma B.10 implies

$$
\left.\left.\limsup _{N \rightarrow \infty} \frac{S_{N}(t)}{N^{1 / 2-2\left(a_{0} \wedge a_{1}\right)}} \leq C \times\left|\tau_{1}-t\right|^{-1 / 2} \quad \text { when } \quad \delta \in\right] \frac{1}{2}, \frac{7-4\left(a_{0} \wedge a_{1}\right)}{8-4\left(a_{0} \wedge a_{1}\right)}\right]
$$

and Lemmas B.4, B.6, B.8, B.9 induce

$$
\left.\left.\lim _{N \rightarrow \infty} \frac{\beta_{N}(t)}{N^{1 / 2-2\left(a_{0} \wedge a_{1}\right)}}=0 \quad \text { when } \quad \delta \in\right] \frac{1}{2}, \frac{7-4\left(a_{0} \wedge a_{1}\right)}{8-4\left(a_{0} \wedge a_{1}\right)}\right] .
$$


Indeed, Lemmas B.4, B.6 and Lemma B.8 are respectively valid under the conditions

$$
\delta>1 / 2, \quad \delta<\frac{3-2\left(a_{0} \wedge a_{1}\right)}{4-2\left(a_{0} \wedge a_{1}\right)} \quad \text { and } \quad \delta<\frac{7-4\left(a_{0} \wedge a_{1}\right)}{8-4\left(a_{0} \wedge a_{1}\right)}
$$

But a direct calculation shows that $\frac{1}{2}<\frac{3-2 a}{4-2 a}<\frac{7-4 a}{8-4 a}$, for every $a \in(0,1)$. Thus (23) and (24) are true for every $t \in \mathbb{R}$. Combined with (22) this induces that Relation (17) holds and finishes the proof of the theorem.

\section{A One deterministic lemma related to wavelets}

From now on, for every integers $N \geq 1, j \geq 0, k \in \mathbf{Z}$ and all real $x, \Delta_{N} \Psi_{a, j, k}$ will be the function defined as

$$
\Delta_{N} \Psi_{a, j, k}(x)=\sum_{\ell=0}^{p} d_{\ell} \Psi_{a}\left(2^{j}\left(\left(x+\frac{\ell}{N}\right)-k\right)\right) .
$$

In the sequel, $C>0$ will denote a generic constant that may change from line to line.

Lemma A.1 For every $a \in(0,1)$, every $\delta \in(0,1)$ and every $\beta$ satisfying $\beta+a>5 / 2$, there exists a constant $C>0$ such that when $N^{(1-\delta)}>p / 2$ the inequalities

$$
\sum_{j \in \mathbb{N}} \sum_{k \in \mathbf{Z}, k / 2^{j} \geq \tau_{1}}\left\{2^{-2 j a}\left|\Delta_{N} \Psi_{a, j, k}(x)\right|^{2}\right\} \leq C \times N^{\delta(2 \beta-1)-4}
$$

and

$$
\sum_{j \in \mathbb{N}} \sum_{k \in \mathbf{Z}, k / 2^{j} \leq \tau_{1}}\left\{2^{-2 j a}\left|\Delta_{N} \Psi_{a, j, k}(x)\right|^{2}\right\} \leq C N^{\delta(2 \beta-1)-4} .
$$

respectively hold when $x \in J_{1}^{\delta}(t, N)$ and when $x \in J_{3}^{\delta}(t, N)$.

Proof. The bound (26) means that for any real number $x$ inside the band left to the change time $J_{1}^{\delta}(t, N)$, the contribution of the dyadic numbers $k / 2^{j}$ at the right of the abrupt change is controlled by $C \times N^{\delta(2 \beta-1)-4}$. The bound (27) means that for any real number $x$ at the right of the change time $J_{3}^{\delta}(t, N)$, the contribution of the dyadic numbers at the left is controlled by the same quantity. Clearly, the two proofs are similar. We just prove the first bound.

The key argument is the fast decreasing of the functions $\Psi_{a}$. Since $\Psi_{a} \in \mathcal{S}(\mathbb{R})$, for all $\alpha>1$ there exists a constant $C>0$ such that for every $x \in \mathbb{R}$,

$$
\left|\Psi_{a}(x)\right| \leq C \times(3+|x|)^{-\alpha} .
$$

We call $S(x)$ the left hand side of (26) and we split it into two series : the high frequencies for $j>j_{0}$ denoted $S_{1}(x)$ and the low frequencies for $j \leq j_{0}$ denoted $S_{2}(x)$. Thus we have that

$$
S(x):=\sum_{j \in \mathbb{N}} \sum_{k \in \mathbf{Z}, k / 2^{j} \geq \tau_{1}}\left\{2^{-2 j a}\left|\Delta_{N} \Psi_{a, j, k}(x)\right|^{2}\right\}=S_{1}(x)+S_{2}(x) .
$$


The critical index $j_{0}$ is determined by the inequalities

$$
2^{-\left(j_{0}+1\right)} \leq \frac{1}{N}<2^{-j_{0}} .
$$

We first bound $S_{1}(x)$. Setting

$$
R_{1}(x)=\sum_{j=j_{0}+1}^{\infty} \sum_{k \in \mathbf{Z}, k / 2^{j} \geq \tau_{1}}\left\{2^{-2 j a}\left|\Psi_{a}\left(2^{j} x-k\right)\right|^{2}\right\},
$$

we obtain that

$$
S_{1}(x) \leq 2 \sum_{\ell=0}^{p}\left\{\left|d_{\ell}\right|^{2} R_{1}\left(x+\frac{\ell}{N}\right)\right\}
$$

Then it follows from (28) and (18) that for all $x \in J_{1}^{\delta}(t, N)$,

$$
\begin{aligned}
R_{1}(x) & \leq C \sum_{j=j_{0}+1}^{\infty} \sum_{k \in \mathbf{Z}, k / 2^{j} \geq \tau_{1}}\left\{2^{-2 j a}\left(3+\left|2^{j} x-k\right|\right)^{-2 \alpha}\right\} \\
& \leq C \times \sum_{j=j_{0}+1}^{\infty} \sum_{l \geq 3 \times 2^{j} / N^{\delta}} 2^{-2 j a}(3+l)^{-2 \alpha} \\
& \leq C \times \sum_{j=j_{0}+1}^{\infty} 2^{-2 j a} \int_{3 \times 2^{j} / N^{\delta}}^{\infty}(2+x)^{-2 \alpha} d x \\
& \leq \frac{C N^{2 \delta \alpha}}{3^{2 \alpha}(2 \alpha-1)} \sum_{j=j_{0}+1}^{\infty} 2^{-j(2 a+2 \alpha-1)} \\
& =C \times N^{2 \delta \alpha} \times \frac{2^{-\left(j_{0}+1\right)(2 a+2 \alpha-1)}}{1-2^{-2 a-2 \alpha+1}}
\end{aligned}
$$

Next, since $a>0$, by using (30), we obtain that for every $x \in J_{1}^{\delta}(t, N)$

$$
R_{1}(x) \leq C \times N^{-2(a+\alpha-\delta \alpha-1 / 2)} .
$$

Next, as the choice of the parameter $\alpha$ remains free, we can impose that $a+\alpha-\delta \alpha-1 / 2 \geq 1$ (or equivalently $\alpha \geq(3 / 2-a) /(1-\delta)$ ), and we get that for every $x \in J_{1}^{\delta}(t, N)$

$$
R_{1}(x) \leq C \times N^{-2}
$$

When $x \leq \tau_{1}-3 N^{-\delta}$ and $y=x+\ell / N$ where $\ell \in\{0,1, \ldots, p\}$, we get for $k / 2^{j} \geq \tau_{1}$

$$
\left|y-k 2^{-j}\right|=\left|x+\ell / N-k 2^{-j}\right| \geq\left|x-k 2^{-j}\right|-\frac{\ell}{N} \geq 3 N^{-\delta}-\frac{\ell}{N} .
$$

Thus

$$
2^{j}\left|y-k 2^{-j}\right| \geq 2^{j} \times 3 N^{-\delta} \times\left(1-\frac{\ell}{3 N^{(1-\delta)}}\right) .
$$


The same calculation as above leads to

$$
R_{1}\left(x+\frac{\ell}{N}\right) \leq C \times\left(1-\frac{\ell}{3 N^{(1-\delta)}}\right)^{-2 \alpha} \times N^{-2} .
$$

The assumption $N^{(1-\delta)}>p / 2$ implies that $1-\frac{p}{3 N^{(1-\delta)}} \geq \frac{1}{3}$. Thus we have

$$
\left[1-\frac{\ell}{3 N^{(1-\delta)}}\right]^{-2 \alpha} \leq 9^{\alpha} \quad \text { for all } \ell=0, \ldots, p .
$$

From (31) and (32) we deduce that for all $x \in J_{1}^{\delta}(t, N)$

$$
S_{1}(x) \leq C \times N^{-2}
$$

It remains to bound $S_{2}(x)$. By using Taylor Formula it follows that, for every $x \in \mathbb{R}$ there exists a $\theta_{x} \in\left(x, x+\frac{p}{N}\right)$ which satisfies

$$
\left|\Delta_{N} \Psi_{a, j, k}(x)\right|=\left|\Psi_{a}^{\prime \prime}\left(2^{j} \theta_{x}-k\right)\right| \times 2^{2 j} N^{-2} .
$$

Since $\Psi_{a} \in \mathcal{S}(\mathbb{R})$, there exists a constant $C>0$ (depending on $p$ ) such that for all $y \in \mathbb{R}$,

$$
\left|\Psi_{a}^{\prime \prime}(y)\right| \leq C \times(2+p+|y|)^{-\beta}
$$

which implies that

$$
\left|\Psi_{a}^{\prime \prime}\left(2^{j} \theta_{x}-k\right)\right| \leq C \times\left(2+p+\left|2^{j} \theta_{x}-k\right|\right)^{-\beta}
$$

Next by (30), we have that $\frac{2^{j} p}{N} \leq p$ for all $j \leq j_{0}$. Then by using the triangular inequality, we get

$$
\left|2^{j} x-k\right| \leq\left|2^{j} \theta_{x}-k\right|+2^{j} \frac{p}{N} \leq\left|2^{j} \theta_{x}-k\right|+p .
$$

Since $\beta>5 / 2-a>0$, we deduce

$$
\left|\Psi_{a}^{\prime \prime}\left(2^{j} \theta_{x}-k\right)\right| \leq C \times\left(2+\left|2^{j} x-k\right|\right)^{-\beta} \quad \text { for all } x \in \mathbb{R}
$$

and then (33) entails that

$$
\left|\Delta_{N} \Psi_{a, j, k}(x)\right| \leq 2^{2 j} N^{-2}\left(2+\left|2^{j} x-k\right|\right)^{-\beta} \quad \text { for all } x \in \mathbb{R} .
$$

Thus for every $x \in J_{1}^{\delta}(t, N)$

$$
\begin{aligned}
\sum_{k \in \mathbf{Z}, k / 2^{j}>\tau_{1}}\left|\Delta_{N} \Psi_{a, j, k}(x)\right|^{2} & \leq 2^{4 j} N^{-4} \sum_{k \in \mathbf{Z}, k / 2^{j}>\tau_{1}}\left(2+\left|k-2^{j} x\right|\right)^{-2 \beta} \\
& \leq 2^{4 j} N^{-4} \int_{3 \times 2^{j} / N^{\delta}}^{\infty}(2+u)^{-2 \beta} d u \\
& =C \times 2^{4 j} N^{-4}\left(2+3 \times 2^{j} \times N^{-\delta}\right)^{-(2 \beta-1)} \\
& \leq C \times 2^{-j(2 \beta-5)} N^{\delta(2 \beta-1)-4}
\end{aligned}
$$


At least, we obtain that for every $x \in J_{1}^{\delta}(t, N)$

$$
\begin{aligned}
S_{2}(x) & \leq \sum_{j \leq j_{0}}\left\{2^{-2 j a} \times C \times 2^{-j(2 \beta-5)} N^{\delta(2 \beta-1)-4}\right\} \\
& =C \times N^{\delta(2 \beta-1)-4} \times\left(\sum_{j \leq j_{0}} 2^{-j(2 \beta+2 a-5)}\right) .
\end{aligned}
$$

By assumption, we have $2 \beta+2 a-5>0$ which implies $S_{2}(x) \leq C \times N^{\delta(\beta-1)-2}$ for all $x \in J_{1}^{\delta}(t, N)$. This finishes the proof of Lemma A.1.

\section{B Some probabilistic lemmas}

Several terms behave like the corresponding ones for the FBM. We give some definitions in force in this section. Let

$$
B_{a}(t)=\sum_{j \in \mathbb{N}} \sum_{k \in \mathbf{Z}} 2^{-j a}\left[\Psi_{a}\left(2^{j} t-k\right)-\Psi_{a}(-k)\right] \zeta_{j, k}+\widetilde{B}_{a}(t)
$$

where $\widetilde{B}$ is defined by $(3)$. The process $\left(B_{a}(t), t>0\right)$ is a FBM with Hurst parameter $a$. For any $a \in(0,1)$, we denote $Y_{a, N}(k)$, resp. $\widetilde{Y}_{a, N}(k)$, the increments defined by (12) when $X$ is replaced by $B_{a}$, resp. $\widetilde{B}_{a}$, that is

$$
Y_{a, N}(k)=\sum_{\ell=0}^{p} d_{\ell} B_{a}\left(\frac{k+\ell}{N}\right), \quad \widetilde{Y}_{a, N}(k)=\sum_{\ell=0}^{p} d_{\ell} \widetilde{B}_{a}\left(\frac{k+\ell}{N}\right) \quad \text { for } \quad k \in \mathbf{Z}
$$

and we set $S_{a, N}$ the quantity defined by

$$
S_{a, N}=\sqrt{\operatorname{Var}\left(\sum_{k=1}^{N \gamma}\left|Y_{a, N}(k)\right|^{2}\right)}
$$

Eventually, we denote $\widetilde{G}_{p, q}^{(0)}(N, t)$, resp. $\widetilde{G}_{p, q}^{(1)}(N, t)$, the analog of $G_{p, q}(N, t)$, when $X$ is replaced by $B_{a_{0}}$, resp. $B_{a_{1}}$, namely

$$
\widetilde{G}_{p, q}^{(i)}(N, t)=\max _{n \in I_{p}(t, N)} \sum_{m \in I_{q}(t, N)}\left|\mathbb{E}\left(Y_{a_{i}, N}(n) Y_{a_{i}, N}(m)\right)\right|, \quad \text { for } \quad i=0 \text { or } 1 .
$$

First, we recall some properties of the FBM

Lemma B.1 Assume that conditions (13) are satisfied. For every a $\in(0,1)$, we have the following properties 
(i) Let $Y_{a, N}(k)$ be defined by (36), then for every $k \in \mathbf{Z}, Y_{a, N}(k)$ is a centered Gaussian r.v. with variance

$$
\mathbb{E}\left(\left|Y_{a, N}(k)\right|^{2}\right)=N^{-2 a} \times C(a, d) \quad \text { where } \quad C(a, d)=\int_{\mathbb{R}}\left|\sum_{\ell=0}^{p} d_{\ell} e^{i \ell u}\right|^{2} \times u^{-(2 a+1)} d u<\infty .
$$

(ii) Let $S_{a, N}$ be defined by (37) and assume conditions (13) are satisfied, then there exist two positive constants $C_{1}, C_{2}$ such that

$$
C_{1} \times N^{1 / 2-2 a} \leq S_{a, N} \leq C_{2} \times N^{1 / 2-2 a} .
$$

(iii) Let $\widetilde{G}_{p, q}^{(i)}(N, t)$ be defined by (38), we have

$$
\widetilde{G}_{p, q}^{(i)}(N, t)=o\left(N^{1 / 2-2 a_{i}}\right) \quad \text { for } \quad i=0 \text { or } 1 .
$$

Remark B.1 The condition of nullity of the second moment of $d$ in (13) is crucial to obtain the upper bound in (39) and derive (40). Indeed, this condition implies that $g_{\mathbf{d}}(v)=\mathcal{O}\left(v^{2}\right)$ where $g_{\mathbf{d}}$ is the function defined by (41). This is explained at the end of the proof of part (ii) of Lemma B.1.

Proof. In this proof, we denote

$$
g_{\mathbf{d}}(v)=\sum_{\ell=0}^{p} d_{\ell} e^{i \ell v}
$$

where $\mathbf{d}$ is the finite sequence associated to the generalized quadratic variation $V_{N}^{(d)}$. The two following properties play a key role in both proof of point (i) and (ii).

- The function $g_{\mathbf{d}}$ and its derivative $g_{\mathbf{d}}^{\prime}$ are bounded on $\mathbb{R}$.

- The conditions (13) are equivalent to $g_{\mathbf{d}}(v)=\mathcal{O}\left(v^{2}\right)$ as $v$ goes to 0 .

Indeed, $\left|e^{i \ell v}\right|=1$ implies the first point. The nullity of the first moment of $d$ implies $g_{\mathbf{d}}(0)=0$ and the nullity of the second one provides $g_{\mathbf{d}}^{\prime}(0)=0$, this induces the second point. With this notation, we have

$$
C(a, d)=\int_{\mathbb{R}}\left|g_{\mathbf{d}}(v)\right|^{2} \times u^{-(2 a+1)} d v
$$

and $C(a, d)<\infty$ as soon as the first moment of $d$ is null, that is $\sum_{\ell=0}^{p} d_{\ell}=0$. We turn us now to the proof of (i), (ii) and (iii).

i) By using conditions $(1,36)$, we get

$$
Y_{a, N}(k)=\int_{\mathbb{R}}\left(\sum_{\ell=0}^{p} d_{\ell} \times e^{i\left(\frac{k+\ell}{N}\right) \xi}\right) \times \frac{|\xi|^{-(a-1 / 2)}}{i \xi} \widehat{W}(\xi) .
$$


Thus

$$
\begin{aligned}
\mathbb{E}\left(\left|Y_{a, N}(k)\right|^{2}\right) & =\int_{\mathbb{R}}\left|\left(\sum_{\ell=0}^{p} d_{\ell} \times e^{i\left(\frac{k+\ell}{N}\right) \xi}\right) \times\left(\frac{|\xi|^{-(a-1 / 2)}}{i \xi}\right)\right|^{2} d \xi \\
& =\int_{\mathbb{R}}\left|\left(\sum_{\ell=0}^{p} d_{\ell} \times e^{i \ell \xi / N}\right)\right|^{2} \times|\xi|^{-(2 a+1)} d \xi \\
& =\int_{\mathbb{R}}\left|g_{\mathbf{d}}\left(\frac{\xi}{N}\right)\right|^{2} \times|\xi|^{-(2 a+1)} d \xi \\
& =N^{-2 a} \times C(a, d),
\end{aligned}
$$

where the last equality results from the change of variable $v=\xi / N$. But Conditions (13) imply that $C(a, d)<\infty$ for all $a \in(0,1)$. Therefore $Y_{a, N}(k)$ is a Gaussian centered r.v. with variance $N^{-2 a} \times C(a, d)$.

ii) Since $S_{a, N}$ corresponds to the $(d)$ quadratic variation of the FBM $B_{a}$ which has stationary increments, the quantities $\sum_{k=[N t]+1}^{[N t]+N \gamma}\left|Y_{a, N}(k)\right|^{2}$ do not depend on the time $t$. Therefore, we could set $t=0$ as it is done in (37). The proof mimics the one given in Bardet (2000). We just gives the main lines. Since the $Y_{a, N}(k)$ are centred Gaussian r.v. we have

$$
\begin{aligned}
S_{a, N}^{2} & =\operatorname{Var}\left(\sum_{k=1}^{N \gamma}\left|Y_{a, N}(k)\right|^{2}\right) \\
& =\sum_{k=1}^{N \gamma} \sum_{m=1}^{N \gamma} \operatorname{Cov}\left(Y_{a, N}(k)^{2}, Y_{a, N}(m)^{2}\right) \\
& =2 \sum_{k=1}^{N \gamma} \sum_{m=1}^{N \gamma} \operatorname{Cov}\left(Y_{a, N}(k), Y_{a, N}(m)\right)^{2} \\
& =2 \sum_{k=1}^{N \gamma} \sum_{m=1}^{N \gamma} \mathbb{E}\left(Y_{a, N}(k) \overline{Y_{a, N}(m)}\right)^{2} .
\end{aligned}
$$

By using successively (42) and the change of variable $v=\xi / N$, we get

$$
\begin{aligned}
\mathbb{E}\left(Y_{a, N}(k) \overline{Y_{a, N}(m)}\right) & =\int_{\mathbb{R}}|\xi|^{-(2 a+1)} \times e^{i\left(\frac{k-m}{N}\right) \xi} \times\left|\sum_{\ell=0}^{p} d_{\ell} \times e^{i\left(\frac{\ell}{N}\right) \xi}\right|^{2} d \xi \\
& =2 N^{-2 a} \int_{\mathbb{R}^{+}} v^{-(2 a+1)} \times \cos ((k-m) v) \times\left|g_{\mathbf{d}}(v)\right|^{2} d v
\end{aligned}
$$

We denote $\rho_{a}(k)=\int_{0}^{\infty} v^{-(2 a+1)} \times \cos (k v) \times\left|g_{\mathbf{d}}(v)\right|^{2} d v$ for $k \in \mathbf{Z}$ and $a \in(0,1)$. Thus we have

$$
S_{a, N}^{2}=4 N^{-4 a} \sum_{k=1}^{N \gamma} \sum_{m=1}^{N \gamma} \rho_{a}(k-m)^{2}
$$


Remark that $\rho_{a}(k)$ is well defined since for every $k \in \mathbf{Z}$ we get $\left|\rho_{a}(k)\right| \leq C(a, d)<\infty$. Moreover we have $\rho_{a}(0)=C(a, d)$. On the one hand, we can bound from below $S_{a, N}^{2}$ by retaining only the diagonal terms in the two series. So, we get

$$
S_{a, N}^{2} \geq 4 N^{-4 a} \sum_{k=m=1}^{N \gamma} \rho_{a}(0)^{2}=C \times N^{1-4 a},
$$

which induces the lower bound of (39). On the other hand, the upper bound follows from technical and tedious calculations. To put in a nutshell, it results from conditions (13) that $\left|g_{\mathbf{d}}(v)\right|^{2}=\mathcal{O}\left(v^{4}\right)$ as $v$ goes to 0 . By using successively two integration by parts (we integrate twice the term $\cos (k v)$ and derive the product term), we get $\left|\rho_{a}(k)\right| \leq C / k^{2}$ for every $k \neq 0$. Thus, we deduce

$$
\begin{aligned}
S_{a, N}^{2} & \leq 4 N^{-4 a}\left[\sum_{k=1}^{N \gamma} \rho_{a}(0)^{2}+\sum_{k=1}^{N \gamma} \sum_{m=1, m \neq k}^{N \gamma} \rho_{a}(k-m)^{2}\right] \\
& \leq C \times N^{1-4 a}+C \times \sum_{k=1}^{N \gamma} \sum_{m=1, m \neq k}^{N \gamma} \frac{1}{|k-m|^{4}} \\
& \leq C \times N^{1-4 a}
\end{aligned}
$$

which implies the upper bound of (39) and finishes the proof of the point (ii).

Let us stress that the upper bound of (39) follows from the bound $\left|\rho_{a}(k)\right| \leq C / k^{2}$ for all integer $k \geq 1$, which results from the nullity of the second moment of $d$, that is $\sum_{\ell=0}^{p} \ell d_{\ell}=0$. Indeed, $\rho_{a}(k)$ is defined as an improper integral both at 0 and $\infty$. Since $\cos (k v)$ and $g_{\mathbf{d}}(v)$ are bounded and $a \in(0,1)$, the term $v^{-(2 a+1)}$ insures the convergence at $\infty$. Similarly, the successive primitives of $\cos (k v)$ and the derivatives of $g_{\mathbf{d}}(v)$ remain bounded, hence the derivatives of $\left|g_{\mathbf{d}}(v)\right|^{2} \times v^{-(2 a+1)}$ would still converge at $\infty$. We focus on the convergence at 0 . Set $f(v)=$ $\left|g_{\mathbf{d}}(v)\right|^{2} \times v^{-(2 a+1)}$. The first integration by parts gives

$$
\int_{0}^{\infty} \cos (k v) \times f(v) d v=-\frac{1}{k} \int_{0}^{\infty} \sin (k v) \times f^{\prime}(v) d v
$$

since $\sin (0)=0$ and $f(\infty)=0$. Then, the second integration provides

$$
\begin{aligned}
\int_{0}^{\infty} \cos (k v) f(v) d v & =-\frac{1}{k^{2}} \int_{0}^{\infty} \cos (k v) \times f^{\prime \prime}(v) d v+\frac{1}{k^{2}}\left[\cos (k v) \times f^{\prime}(v)\right]_{0}^{\infty} \\
& =-\frac{1}{k^{2}} \int_{0}^{\infty} \cos (k v) \times f^{\prime \prime}(v) d v-\frac{1}{k^{2}} f^{\prime}(0)
\end{aligned}
$$

since $f^{\prime}(\infty)=0$ and $\cos (0)=1$. But $g_{\mathbf{d}}(v)=\mathcal{O}\left(v^{2}\right)$ induces $f^{\prime \prime}(v)=v^{1-2 a} \times(C+\mathcal{O}(v))$ as $v$ goes to 0 . This insures the convergence of the integral $\int_{0}^{\infty} \cos (k v) \times f^{\prime \prime}(v) d v$ at 0 , since $1-2 a>-1$ for all $a \in(0,1)$. To conclude with, we remark that if $\sum_{\ell=0}^{p} \ell d_{\ell} \neq 0$ then $f^{\prime \prime}(v)=$ 
$v^{-1-2 a} \times(C+\mathcal{O}(v))$ as $v$ goes to 0 and $\int_{0}^{\infty} \cos (k v) \times f^{\prime \prime}(v) d v=\infty$. In this case, we would have $\left|\rho_{a}(k)\right|=\mathcal{O}(1 / k)$, therefore the upper bound of (39) does not hold.

iii)Denote

$$
\beta_{a, N}(t)=\max _{n \in I_{N}(t)} \sum_{m \in I_{N}(t)}\left|\operatorname{cov}\left(Y_{a_{i}, N}(n), Y_{a_{i}, N}(m)\right)\right| \quad \text { for } \quad i=0 \text { or } 1
$$

From Istas \& Lang, we have $\lim _{N \rightarrow \infty} \beta_{a_{i}, N}(t) / S_{a_{i}, N}=0$. But $\beta_{a_{i}, N} \geq \widetilde{G}_{p, q}^{(i)}(N, t) \geq 0$, for all $p, q \in\{1,2,3\}$. Combined with (39) this induces (40).

Next, we give two Lemmas concerning the regularity in quadratic mean of the high frequencies and low frequencies component of (35).

Lemma B.2 Let $\widetilde{Y}_{a, N}$ be defined by (36). There exists a constant $C>0$ such that for all $a \in(0,1)$ and for every integers $n, m \in I_{N}(t)$,

$$
\left|\mathbb{E}\left(\widetilde{Y}_{a, N}(n) \overline{\widetilde{Y}_{a, N}(m)}\right)\right| \leq C \times N^{-4}
$$

Proof. Since $\varphi$ is a Lemarié-Meyer scale function, we have Supp $\widehat{\varphi} \subset[-4 \pi / 3,4 \pi / 3]$. For every integer $k$, this induces

$$
\int_{\mathbb{R}} \frac{\left(e^{i t \xi}-1\right)}{|\xi|^{a+1 / 2}} e^{-i k \xi} \overline{\widehat{\varphi}(\xi)} d \xi=\int_{0}^{2 \pi}\left(\sum_{r=-\infty}^{\infty} \frac{\left(e^{i t(\xi+2 r \pi)}-1\right)}{|\xi+2 r \pi|^{a+1 / 2}} \overline{\widehat{\varphi}(\xi+2 r \pi)}\right) e^{-i k \xi} d \xi
$$

where the sum is a finite sum, which in fact only contains the terms corresponding to the indices $r=-1$ and $r=0$. Since $\sum_{\ell=0}^{p} d_{\ell}=0$, we have

$$
\tilde{Y}_{a, N}(n)=\sum_{k \in \mathbf{Z}}\left\{\int_{0}^{2 \pi} e^{-i k \xi}\left(\sum_{r=-1}^{0} \frac{\overline{\hat{\varphi}(\xi+2 r \pi)}}{|\xi+2 r \pi|^{a+1 / 2}}\right) \times\left(\sum_{\ell=0}^{p} d_{\ell} e^{i(\xi+2 r \pi)\left(\frac{n+\ell}{N}\right)}\right) d \xi\right\} \eta_{k}
$$

Combined with the Parseval formula, we get

$$
\begin{aligned}
\mathbb{E}\left(\widetilde{Y}_{a, N}(n) \widetilde{\widetilde{Y}}_{a, N}(m)\right)= & \sum_{k \in \mathbf{Z}}\left\{\int_{0}^{2 \pi} e^{-i k \xi}\left(\sum_{r=-1}^{0} \frac{\widehat{\varphi}(\xi+2 r \pi)}{|\xi+2 r \pi|^{a+1 / 2}}\right) \times\left(\sum_{\ell=0}^{p} d_{\ell} e^{i(\xi+2 r \pi)\left(\frac{n+\ell}{N}\right)}\right) d \xi\right\} \\
& \times\left\{\int_{0}^{2 \pi} e^{i k \xi}\left(\sum_{r=-1}^{0} \frac{\bar{\varphi}(\hat{\varphi}(\xi+2 r \pi)}{|\xi+2 r \pi|^{a+1 / 2}}\right) \times\left(\sum_{\ell=0}^{p} d_{\ell} e^{-i(\xi+2 r \pi)\left(\frac{m+\ell}{N}\right)}\right) d \xi\right\} \\
= & \int_{0}^{2 \pi}\left(\sum_{r=-1}^{0} \frac{\widehat{\varphi}(\xi+2 r \pi)}{|\xi+2 r \pi|^{a+1 / 2}}\right) \times\left(\sum_{\ell=0}^{p} d_{\ell} e^{i(\xi+2 r \pi)\left(\frac{n+\ell}{N}\right)}\right) \\
& \times\left(\sum_{r=-1}^{0} \frac{\frac{\hat{\varphi}(\xi+2 r \pi)}{|\xi+2 r \pi|^{a+1 / 2}}}{\mid \xi\left(\sum_{\ell=0}^{p} d_{\ell} e^{-i(\xi+2 r \pi)\left(\frac{m+\ell}{N}\right)}\right)}\right) d \xi .
\end{aligned}
$$


From the other hand, by using Relations (13) and Taylor formula, one obtains that for every $\theta \in \mathbb{R}$

$$
\left|\sum_{\ell=0}^{p} d_{\ell} e^{i \ell \theta}\right| \leq \frac{1}{2}|\theta|^{2} \times\left(\sum_{\ell=0}^{p} \ell^{2}\left|d_{\ell}\right|\right)
$$

which induces

$$
\left|\sum_{\ell=0}^{p} d_{\ell} e^{i(\xi+2 r \pi)\left(\frac{m+\ell}{N}\right)}\right| \leq \frac{1}{N^{2}} \times|\xi+2 r \pi|^{2} \times \frac{1}{2}\left(\sum_{\ell=0}^{p} \ell^{2}\left|d_{\ell}\right|\right) .
$$

Since $\widehat{\varphi}$ is a bounded function, we deduce the following upper bound

$$
\begin{aligned}
\left|\mathbb{E}\left(\widetilde{Y}_{a, N}(n) \overline{\widetilde{Y}_{a, N}(m)}\right)\right| \leq & \frac{1}{N^{4}} \times\|\widehat{\varphi}\|_{L^{\infty}}^{2} \times \frac{1}{4}\left(\sum_{\ell=0}^{p} \ell^{2}\left|d_{\ell}\right|\right)^{2} \\
& \times \int_{0}^{2 \pi}\left(\sum_{r=-1}^{0}|\xi+2 r \pi|^{3 / 2-a}\right)^{2} d \xi
\end{aligned}
$$

which implies (43), since $3 / 2-a \in] 1 / 2,3 / 2[$.

Lemma B.3 For every $a \in(0,1)$ we have

$$
\sum_{j \in \mathbb{N}} \sum_{k \in \mathbf{Z}} 2^{-2 j a}\left|\Delta_{N} \Psi_{a, j, k}\left(\frac{n}{N}\right)\right|^{2} \leq C \times N^{-2 a}+C \times N^{-4} .
$$

Proof. From $(7,12,25,35,36)$, we have

$$
\begin{aligned}
\sum_{j \in \mathbb{N}} \sum_{k \in \mathbf{Z}} 2^{-2 j a}\left|\Delta_{N} \Psi_{a, j, k}\left(\frac{n}{N}\right)\right|^{2} & =\mathbb{E}\left(\left|Y_{a, N}(n)-\tilde{Y}_{a, N}(n)\right|^{2}\right) \\
& \leq 2 \mathbb{E}\left(\left|Y_{a, N}(n)\right|^{2}\right)+2 \mathbb{E}\left(\left|\widetilde{Y}_{a, N}(n)\right|^{2}\right) \\
& =C \times N^{-2 a}+C \times N^{-4}
\end{aligned}
$$

where the last equality results from Lemma B.1 (i) and Lemma B.2.

We deduce the asymptotic bound on $F(t, N)$ :

Lemma B.4 Let $F(t, N)$ be defined by (20). For every $\delta \in(1 / 2,1)$ we have

$$
\lim _{N \rightarrow \infty} \frac{F(t, N)}{N^{1 / 2-2\left(a_{0} \wedge a_{1}\right)}}=0 .
$$

Proof. From $(7,9,12,25)$ combined with Lemma B.3, we get

$$
\mathbb{E}\left(\left|Y_{N}(n)\right|^{2}\right)=\sum_{j \in \mathbb{N}} \sum_{k \in \mathbf{Z}, k / 2^{j}<\tau_{1}} 2^{-2 j a_{0}}\left|\Delta_{N} \Psi_{a_{0}, j, k}\left(\frac{n}{N}\right)\right|^{2}
$$




$$
\begin{aligned}
& +\sum_{j \in \mathbb{N}} \sum_{k \in \mathbf{Z}, k / 2^{j} \geq \tau_{1}} 2^{-2 j a_{1}}\left|\Delta_{N} \Psi_{a_{1}, j, k}\left(\frac{n}{N}\right)\right|^{2} \\
\leq & \sum_{j \in \mathbb{N}} \sum_{k \in \mathbf{Z}} 2^{-2 j a_{0}}\left|\Delta_{N} \Psi_{a_{0}, j, k}\left(\frac{n}{N}\right)\right|^{2}+\sum_{j \in \mathbb{N}} \sum_{k \in \mathbf{Z}} 2^{-2 j a_{1}}\left|\Delta_{N} \Psi_{a_{1}, j, k}\left(\frac{n}{N}\right)\right|^{2} \\
\leq & C \times N^{-2 a_{0}}+C \times N^{-2 a_{1}}+C \times N^{-4} .
\end{aligned}
$$

From Cauchy-Schwarz inequality, we get

$$
\left|\mathbb{E}\left(Y_{N}(n) Y_{N}(m)\right)\right| \leq \sqrt{\mathbb{E}\left(Y_{N}(n)^{2}\right)} \times \sqrt{\mathbb{E}\left(Y_{N}(m)^{2}\right)}
$$

This induces

$$
F(t, N) \leq \operatorname{Card}_{2}(t, N) \times\left(C \times N^{-2 a_{0}}+C \times N^{-2 a_{1}}+C \times N^{-4}\right)
$$

By definition, we have $\operatorname{Card} I_{2}(t, N) \leq 6 N^{1-\delta}$. By using $\delta>1 / 2$, we deduce

$$
F(t, N) \leq C \times N^{1-\delta-2\left(a_{0} \wedge a_{1}\right)}=o\left(N^{1 / 2-2\left(a_{0} \wedge a_{1}\right)}\right) .
$$

The asymptotic behavior of $G_{1,1}$ and $G_{3,3}$ is given by Lemma B.7 which proof needs the following Lemma:

Lemma B.5 Let $Y_{a_{0}, N}$ and $Y_{a_{1}, N}$ be defined by (36). Then for all $\beta$ such that $\beta+a_{0}>5 / 2$ and $\beta+a_{1}>5 / 2$, for every $\delta \in(0,1)$ there exists a constant $C>0$ such that

$$
\begin{array}{ll}
\forall n, m \in I_{1}^{\delta}(t, N) & \left|\mathbb{E}\left(Y_{N}(n) Y_{N}(m)\right)-\mathbb{E}\left(Y_{a_{0}, N}(n) Y_{a_{0}, N}(m)\right)\right| \leq C \times N^{\delta(2 \beta-1)-4} \\
\forall n, m \in I_{3}^{\delta}(t, N) & \left|\mathbb{E}\left(Y_{N}(n) Y_{N}(m)\right)-\mathbb{E}\left(Y_{a_{1}, N}(n) Y_{a_{1}, N}(m)\right)\right| \leq C \times N^{\delta(2 \beta-1)-4} .
\end{array}
$$

Proof. The two inequalities can be obtained similarly, so we will only prove the first one. Relations $(7,12,25)$ and (9) imply that

$$
\begin{aligned}
\mathbb{E}\left(Y_{N}(n) Y_{N}(m)\right) & =\sum_{j \in \mathbb{N}} \sum_{k \in \mathbf{Z}, k / 2^{j}<\tau_{1}} 2^{-2 j a_{0}} \Delta_{N} \Psi_{a_{0}, j, k}\left(\frac{n}{N}\right) \times \Delta_{N} \Psi_{a_{0}, j, k}\left(\frac{m}{N}\right) \\
& +\sum_{j \in \mathbb{N}} \sum_{k \in \mathbf{Z}, k / 2^{j} \geq \tau_{1}} 2^{-2 j a_{1}} \Delta_{N} \Psi_{a_{1}, j, k}\left(\frac{n}{N}\right) \times \Delta_{N} \Psi_{a_{1}, j, k}\left(\frac{m}{N}\right)
\end{aligned}
$$

From the another hand, we have that for $i=0$ or 1

$$
\begin{aligned}
\mathbb{E}\left(Y_{a_{i}, N}(n) Y_{a_{i}, N}(m)\right) & =\mathbb{E}\left(Y_{a_{i}, N}(n) \overline{Y_{a_{i}, N}(m)}\right) \\
& =\sum_{j \in \mathbb{N}} \sum_{k \in \mathbf{Z}} 2^{-2 j a_{i}} \Delta_{N} \Psi_{a_{i}, j, k}\left(\frac{n}{N}\right) \times \Delta_{N} \Psi_{a_{i}, j, k}\left(\frac{m}{N}\right) \\
& +\mathbb{E}\left(\widetilde{Y}_{a_{i}, N}(n) \overline{\widetilde{Y}}_{a_{i}, N}(m)\right)
\end{aligned}
$$


where $\widetilde{Y}_{a_{i}, N}(k)=\sum_{\ell=0}^{p} d_{\ell} \widetilde{B}_{a_{i}}\left(\frac{k+\ell}{N}\right)$. Combined with Lemma B.2, we get

$$
\begin{aligned}
\mid \mathbb{E}\left(Y_{N}(n) Y_{N}(m)\right. & \left.-Y_{a_{0}, N}(n) Y_{a_{0}, N}(m)\right) \mid \leq C N^{-4} \\
& +\sum_{j \in \mathbb{N}} \sum_{k \in \mathbf{Z}, k / 2^{j} \geq \tau_{1}} 2^{-2 j a_{0}}\left|\Delta_{N} \Psi_{a_{0}, j, k}\left(\frac{n}{N}\right) \Delta_{N} \Psi_{a_{0}, j, k}\left(\frac{m}{N}\right)\right| \\
& +\sum_{j \in \mathbb{N}} \sum_{k \in \mathbf{Z}, k / 2^{j} \geq \tau_{1}} 2^{-2 j a_{1}}\left|\Delta_{N} \Psi_{a_{1}, j, k}\left(\frac{n}{N}\right) \Delta_{N} \Psi_{a_{1}, j, k}\left(\frac{m}{N}\right)\right| .
\end{aligned}
$$

Then Cauchy-Schwarz inequality implies that for $i=0$ or 1

$$
\begin{aligned}
& \sum_{j \in \mathbb{N}} \sum_{k \in \mathbf{Z}, k / 2^{j} \geq \tau_{1}} 2^{-2 j a_{i}}\left|\Delta_{N} \Psi_{a_{i}, j, k}\left(\frac{n}{N}\right) \Delta_{N} \Psi_{a_{i}, j, k}\left(\frac{m}{N}\right)\right| \\
& \leq\left(\sum_{j \in \mathbb{N}} \sum_{k \in \mathbf{Z}, k / 2^{j} \geq \tau_{1}} 2^{-2 j a_{i}}\left|\Delta_{N} \Psi_{a_{i}, j, k}\left(\frac{n}{N}\right)\right|^{2}\right)^{1 / 2} \\
& \times\left(\sum_{j \in \mathbb{N}} \sum_{k \in \mathbf{Z}, k / 2^{j} \geq \tau_{1}} 2^{-2 j a_{i}}\left|\Delta_{N} \Psi_{a_{i}, j, k}\left(\frac{m}{N}\right)\right|^{2}\right)^{1 / 2} \\
& \leq C \times N^{\delta(2 \beta-1)-4},
\end{aligned}
$$

where the last inequality follows from lemma A.1 and holds for $n, m \in I_{1}^{\delta}(t, N)$ and $\beta+a_{i}>5 / 2$. We deduce that all for $n, m \in I_{1}^{\delta}(t, N)$

$$
\left|\mathbb{E}\left(Y_{N}(n) Y_{N}(m)-Y_{a_{0}, N}(n) Y_{a_{0}, N}(m)\right)\right| \leq C \times N^{\delta(2 \beta-1)-4}+C \times N^{-4} .
$$

This finishes the proof of the first bound of Lemma B.5. Mutatis mutandis, the proof of the second bound is the same.

Lemma B.6 Let $G_{1,1}(N, t)$ and $G_{3,3}(N, t)$ be defined by (19).

$$
\text { If } \delta<\frac{7-4\left(a_{0} \wedge a_{1}\right)}{8-4\left(a_{0} \wedge a_{1}\right)}, \quad \text { then } \quad \lim _{N \rightarrow \infty} \frac{G_{1,1}(N, t)}{N^{1 / 2-2\left(a_{0} \wedge a_{1}\right)}}=0 \quad \text { and } \quad \lim _{N \rightarrow \infty} \frac{G_{3,3}(N, t)}{N^{1 / 2-2\left(a_{0} \wedge a_{1}\right)}}=0 \text {. }
$$

Proof. For sake of simplicity, we assume that $a_{0}<a_{1}$. The idea behind the proof is that the covariance of the increments mimics the corresponding one for a FBM when both indices are on the same part of the change point. More precisely, with the same notations as in (38), we have

$$
\begin{aligned}
G_{1,1}(N, t) & \leq \widetilde{G}_{1,1}^{(0)}(N, t)+\max _{n \in I_{1}(t, N)} \sum_{m \in I_{1}(t, N)}\left|\mathbb{E}\left(Y_{N}(n) Y_{N}(m)-Y_{0, N}(n) Y_{0, N}(m)\right)\right| \\
G_{3,3}(N, t) & \leq \widetilde{G}_{3,3}^{(1)}(N, t)+\max _{n \in I_{3}(t, N)} \sum_{m \in I_{3}(t, N)}\left|\mathbb{E}\left(Y_{N}(n) Y_{N}(m)-Y_{1, N}(n) Y_{1, N}(m)\right)\right|
\end{aligned}
$$


From Lemma B.5, for every $\beta>5 / 2-a_{0}$, we have

$$
\begin{aligned}
& G_{1,1}(N, t) \leq \widetilde{G}_{1,1}^{(0)}(N, t)+\operatorname{Card} I_{1}^{\delta}(t, N) \times C \times N^{\delta(2 \beta-1)-4} \\
& G_{3,3}(N, t) \leq \widetilde{G}_{3,3}^{(1)}(N, t)+\operatorname{Card} I_{3}^{\delta}(t, N) \times C \times N^{\delta(2 \beta-1)-4} .
\end{aligned}
$$

From Lemma B.1, we have $\widetilde{G}_{p, q}^{(i)}(N, t)=o\left(N^{1 / 2-2 a_{i}}\right)$ for $i=0$ or 1 . Moreover as $I_{p}^{\delta}(t, N) \subset$ $I_{N}(t)$, we get that $\operatorname{Card} I_{p}^{\delta}(t, N) \leq \operatorname{Card} I_{N}(t) \leq N \gamma$ for $p=1,2$ or 3 . This induces

$$
\begin{aligned}
\frac{G_{1,1}(N, t)}{N^{1 / 2-2\left(a_{0} \wedge a_{1}\right)}} \leq o(1)+C \times N^{\delta(2 \beta-1)+2 a_{0}-7 / 2}, \\
\frac{G_{3,3}(N, t)}{N^{1 / 2-2\left(a_{0} \wedge a_{1}\right)}} \leq o\left(N^{-2\left(a_{1}-a_{0}\right)}\right)+C \times N^{\delta(2 \beta-1)+2 a_{0}-7 / 2} .
\end{aligned}
$$

Since $a_{0}<a_{1}$, the first term of the right hand side of the second inequality converges to 0 . Therefore Lemma B.6 results from the existence of a real number $\beta$ satisfying $\beta>5 / 2-a_{0}$ and $\delta(2 \beta-1)+2 a_{0}-7 / 2<0$, which is equivalent to the condition $\delta\left(4-2 a_{0}\right)<7 / 2-2 a_{0}$. This finishes the proof of Lemma B.6.

The asymptotic behavior of the quantities $G_{1,3}(N, t)$ and $G_{3,1}(N, t)$ is given in Lemma B.8 which will be a consequence of following result :

Lemma B.7 Let $Y_{N}$ be defined by (12). Then for all $\beta$ such that $\beta+\left(a_{0} \wedge a_{1}\right)>5 / 2$, for every $\delta \in(0,1)$ there exists a constant $C>0$ such that if $(n, m) \in I_{1}^{\delta}(t, N) \times I_{3}^{\delta}(t, N)$ then

$$
\left|\mathbb{E}\left(Y_{N}(n) Y_{N}(m)\right)\right| \leq C \times N^{\delta(\beta-1 / 2)-2-\left(a_{0} \wedge a_{1}\right)} .
$$

Proof. Formula (46) is valid for every pair $(n, m) \in \mathbf{Z}^{2}$. From the Cauchy-Schwarz inequality, a straightforward calculation gives us

$$
\begin{aligned}
& \left|\mathbb{E}\left(Y_{N}(n) Y_{N}(m)\right)\right| \\
\leq & \left(\sum_{j \in \mathbb{N}} \sum_{k \in \mathbf{Z}} 2^{-2 j a_{0}}\left|\Delta_{N} \Psi_{a_{0}, j, k}\left(\frac{n}{N}\right)\right|^{2}\right)^{1 / 2} \times\left(\sum_{j \in \mathbb{N}} \sum_{k \in \mathbf{Z}, k / 2^{j}<\tau_{1}} 2^{-2 j a_{0}}\left|\Delta_{N} \Psi_{a_{0}, j, k}\left(\frac{m}{N}\right)\right|^{2}\right)^{1 / 2} \\
+ & \left(\sum_{j \in \mathbb{N}} \sum_{k \in \mathbf{Z}, k / 2^{j} \geq \tau_{1}} 2^{-2 j a_{1}}\left|\Delta_{N} \Psi_{a_{1}, j, k}\left(\frac{n}{N}\right)\right|^{2}\right)^{1 / 2} \times\left(\sum_{j \in \mathbb{N}} \sum_{k \in \mathbf{Z}} 2^{-2 j a_{1}}\left|\Delta_{N} \Psi_{a_{1}, j, k}\left(\frac{m}{N}\right)\right|^{2}\right)^{1 / 2}
\end{aligned}
$$

Since $n / N \in J_{1}^{\delta}(t, N)$ and $m / N \in J_{3}^{\delta}(t, N)$, from Lemma A.1, we get

$$
\begin{aligned}
& \left|\mathbb{E}\left(Y_{N}(n) Y_{N}(m)\right)\right| \leq C N^{\delta(\beta-1 / 2)-2} \\
& \quad \times\left\{\left(\sum_{j \in \mathbb{N}} \sum_{k \in \mathbf{Z}} 2^{-2 j a_{0}}\left|\Delta_{N} \Psi_{a_{0}, j, k}\left(\frac{n}{N}\right)\right|^{2}\right)^{1 / 2}+\left(\sum_{j \in \mathbb{N}} \sum_{k \in \mathbf{Z}} 2^{-2 j a_{1}}\left|\Delta_{N} \Psi_{a_{1}, j, k}\left(\frac{m}{N}\right)\right|^{2}\right)^{1 / 2}\right\} .
\end{aligned}
$$


From Lemma B.3, we get

$$
\left|\mathbb{E}\left(Y_{N}(n) Y_{N}(m)\right)\right| \leq C N^{\delta(\beta-1 / 2)-2} \times\left\{C \times N^{-a_{0}}+C \times N^{-a_{1}}\right\}
$$

which implies (48) and finishes the proof of Lemma B.7.

Lemma B.8 Let $G_{1,3}(N, t)$ and $G_{3,1}(N, t)$ be defined by (19).

If $\delta<\frac{3-2\left(a_{0} \wedge a_{1}\right)}{4-2\left(a_{0} \wedge a_{1}\right)} \quad$ then $\quad \lim _{N \rightarrow \infty} \frac{G_{1,3}(N, t)}{N^{1 / 2-2\left(a_{0} \wedge a_{1}\right)}}=0 \quad$ and $\quad \lim _{N \rightarrow \infty} \frac{G_{3,1}(N, t)}{N^{1 / 2-2\left(a_{0} \wedge a_{1}\right)}}=0$.

Proof. To fix the idea, we suppose $a_{0}<a_{1}$. From $(39,48)$, for all $\beta$ such that $\beta+a_{0}>5 / 2$, we have

$$
\begin{aligned}
& G_{1,3}(N, t) \leq \operatorname{Card} I_{1}^{\delta}(t, N) \times C N^{\delta(\beta-1 / 2)-2-a_{0}} \\
& \text { and } \quad G_{3,1}(N, t) \leq \operatorname{Card} I_{3}^{\delta}(t, N) \times C N^{\delta(\beta-1 / 2)-2-a_{0}}
\end{aligned}
$$

Since $\operatorname{Card} I_{1}^{\delta}(t, N) \leq N \gamma$ and $\operatorname{Card} I_{3}^{\delta}(t, N) \leq N \gamma$, we get

$$
\frac{G_{1,3}(N, t)}{N^{1 / 2-2 a_{0}}} \leq C \times N^{\delta(\beta-1 / 2)-3 / 2+a_{0}} \quad \text { and } \quad \frac{G_{3,1}(N, t)}{N^{1 / 2-2 a_{0}}} \leq C \times N^{\delta(\beta-1 / 2)-3 / 2+a_{0}} .
$$

Therefore Lemma B.8 follows from the existence of a real number $\beta$ satisfying $(\beta-1 / 2)>\left(2-a_{0}\right)$ and $\delta(\beta-1 / 2)-3 / 2+a_{0}<0$, which is equivalent to the condition $\delta\left(2-a_{0}\right)<\left(3 / 2-a_{0}\right)$ assumed in the assumption of Lemma B.8.

Lemma B.9 Let $G_{2,1}(N, t)$ and $G_{2,3}(N, t)$ be defined by (19).

$$
\text { If } \delta<\frac{3-2\left(a_{0} \wedge a_{1}\right)}{4-2\left(a_{0} \wedge a_{1}\right)} \quad \text { then } \quad \lim _{N \rightarrow \infty} \frac{G_{2,1}(N, t)}{N^{1 / 2-2\left(a_{0} \wedge a_{1}\right)}}=0 \quad \text { and } \quad \lim _{N \rightarrow \infty} \frac{G_{2,3}(N, t)}{N^{1 / 2-2\left(a_{0} \wedge a_{1}\right)}}=0 .
$$

Proof. To fix the idea, we suppose $a_{0}<a_{1}$. With the notations defined by (38), we have

$$
\begin{aligned}
& G_{2,1}(N, t) \leq \widetilde{G}_{2,1}^{(0)}(N, t)+\max _{n \in I_{2}(t, N)} \sum_{m \in I_{1}(t, N)}\left|\mathbb{E}\left(Y_{N}(n) Y_{N}(m)-Y_{a_{0}, N}(n) Y_{a_{0}, N}(m)\right)\right|, \\
& G_{2,3}(N, t) \leq \widetilde{G}_{2,3}^{(1)}(N, t)+\max _{n \in I_{2}(t, N)} \sum_{m \in I_{3}(t, N)}\left|\mathbb{E}\left(Y_{N}(n) Y_{N}(m)-Y_{a_{1}, N}(n) Y_{a_{1}, N}(m)\right)\right| .
\end{aligned}
$$

The bound (47) is valid for every pair $(n, m) \in I_{N}(t)^{2}$. By using successively Lemma A.1 and Lemma B.3, we get for $i=0$ or 1

$$
\begin{aligned}
\left|\mathbb{E}\left(Y_{N}(n) Y_{N}(m)-Y_{a_{i}, N}(n) Y_{a_{i}, N}(m)\right)\right| & \leq C N^{-4} \\
& +C \times N^{\delta(\beta-1 / 2)-2} \times\left[C \times N^{-a_{0}}+C \times N^{-a_{1}}\right]
\end{aligned}
$$


for every $\beta$ such that $\beta+a_{0}>5 / 2$. This induces

$$
G_{2,1}(N, t) \leq \widetilde{G}_{2,1}^{(0)}(N, t)+\operatorname{Card} I_{1}^{\delta}(t, N) \times\left[C N^{-4}+N^{\delta(\beta-1 / 2)-2-\left(a_{0} \wedge a_{1}\right)}\right]
$$

From (40) and $\operatorname{Card} I_{1}^{\delta}(t, N) \leq N \gamma$, we deduce

$$
\frac{G_{2,1}(N, t)}{N^{1 / 2-2\left(a_{0} \wedge a_{1}\right)}} \leq o(1)+C \times N^{\delta(\beta-1 / 2)-3 / 2+a_{0}} .
$$

Similar calculations lead to

$$
\frac{G_{2,3}(N, t)}{N^{1 / 2-2 a_{0}}} \leq o\left(N^{-2\left(a_{1}-a_{0}\right)}\right)+C \times N^{\delta(\beta-1 / 2)-3 / 2+a_{0}} .
$$

Therefore Lemma B.9 follows from the existence of a real number $\beta$ satisfying $(\beta-1 / 2)>\left(2-a_{0}\right)$ and $\delta(\beta-1 / 2)-3 / 2+a_{0}<0$, which is equivalent to the condition $\delta\left(2-a_{0}\right)<\left(3 / 2-a_{0}\right)$ assumed in the assumption of Lemma B.9.

Lemma B.10 If $\delta<\frac{7-4\left(a_{0} \wedge a_{1}\right)}{8-4\left(a_{0} \wedge a_{1}\right)}$, then there exists a constant $C>0$ such that

$$
\liminf _{N \rightarrow \infty} \frac{S_{N}(t)}{N^{1 / 2-2\left(a_{0} \wedge a_{1}\right)}} \geq C \times \sqrt{\left|\tau_{1}-t\right|}
$$

Proof. To fix the idea, we assume that $a_{0}<a_{1}$. Since $Y_{N}(n)$ are Gaussain centered r.v., we have

$$
\begin{aligned}
S_{N}(t)^{2}=\operatorname{Var}\left(V_{N}(t)\right) & =\sum_{n \in I_{N}(t)} \sum_{m \in I_{N}(t)} \operatorname{cov}\left(Y_{N}^{2}(n) Y_{N}^{2}(m)\right) \\
& =2 \sum_{n \in I_{N}(t)} \sum_{m \in I_{N}(t)} \operatorname{cov}\left(Y_{N}(n) Y_{N}(m)\right)^{2} \\
& =2 \sum_{n \in I_{N}(t)} \sum_{m \in I_{N}(t)} \mathbb{E}\left(Y_{N}(n) Y_{N}(m)\right)^{2} \\
& \geq 2 \sum_{n \in I_{1}^{\delta}(t, N)} \sum_{m \in I_{1}^{\delta}(t, N)} \mathbb{E}\left(Y_{N}(n) Y_{N}(m)\right)^{2} .
\end{aligned}
$$

Then

$$
\begin{aligned}
\frac{1}{\sqrt{2}} S_{N}(t) \geq & {\left[\sum_{n \in I_{1}^{\delta}(t, N)} \sum_{m \in I_{1}^{\delta}(t, N)} \mathbb{E}\left(Y_{a_{0}, N}(n) Y_{a_{0}, N}(m)\right)^{2}\right]^{1 / 2} } \\
& -\left[\sum_{n \in I_{1}^{\delta}(t, N)} \sum_{m \in I_{1}^{\delta}(t, N)} \mathbb{E}\left(Y_{N}(n) Y_{N}(m)-Y_{a_{0}, N}(n) Y_{a_{0}, N}(m)\right)^{2}\right]^{1 / 2} \\
\geq & {\left[\sum_{n \in I_{1}^{\delta}(t, N)} \mathbb{E}\left(Y_{a_{0}, N}(n)^{2}\right)^{2}\right]^{1 / 2} }
\end{aligned}
$$




$$
-\left[\sum_{n \in I_{1}^{\delta}(t, N)} \sum_{m \in I_{1}^{\delta}(t, N)} \mathbb{E}\left(Y_{N}(n) Y_{N}(m)-Y_{a_{0}, N}(n) Y_{a_{0}, N}(m)\right)^{2}\right]^{1 / 2} .
$$

Next, by using Lemmas B.1 and B.5, for every $\beta$ satisfying $\beta+a_{0}>5 / 2$, we get

$$
\frac{1}{\sqrt{2}} S_{N}(t) \geq \operatorname{Card} I_{1}^{\delta}(t, N)^{1 / 2} \times C \times N^{-2 a_{0}}-C \times C \operatorname{Card} I_{1}^{\delta}(t, N) \times N^{\delta(2 \beta-1)-4}
$$

By using $\operatorname{Card} I_{1}^{\delta}(t, N)$, we deduce

$$
\frac{S_{N}(t)}{N^{1 / 2-2\left(a_{0} \wedge a_{1}\right)}} \geq \sqrt{N^{-1} \times \operatorname{CardI}_{1}^{\delta}(t, N)} \times C-C \times N^{\delta(2 \beta-1)+2 a_{0}-7 / 2}
$$

Since $\delta<\frac{7-4 a_{0}}{8-4 a_{0}}$, there exists a $\beta>5 / 2-a_{0}$ such that $\delta(2 \beta-1)+2 a_{0}-7 / 2<0$. From the other hand, when $t<\tau_{1}<t+\gamma$ we have $N^{-1} \times \operatorname{Card}_{1}^{\delta}(t, N) \rightarrow\left|\tau_{1}-t\right|$ when $N \rightarrow<\infty$. Therefore, we deduce $\liminf _{N \rightarrow \infty} \frac{S_{N}(t)}{N^{1 / 2-2\left(a_{0} \wedge a_{1}\right)}} \geq C \times \sqrt{\left|\tau_{1}-t\right|}$.

Acknowledgment : We would like to thank the anonymous referee for all his valuable comments which have helped us to improve the earlier version of our work.

\section{References}

[1] Ayache, A. and Lévy Véhel, J., "The Generalized Multifractional Brownian Motion." S.I.S.P., Vol. 3, Issue 1/2, p. 7-18, 2000.

[2] Bardet J.M. "Testing for the presence of self-similarity of Gaussian time series having stationary increments." J. of Time Series Anal. 21, p. 497-516, 2000.

[3] Bardet, J.M. and Bertrand P., "Definition, properties and wavelet analysis of multiscale fractional Brownian motion", submitted, 2003.

[4] Barnsley, M.F., Devaney, R.L., Mandelbrot, B.B., Peitgen, H.O., Saupe, D. and Voss, R.F., The science of fractal images, Springer Verlag, 1988.

[5] Benassi, A., Bertrand, P., Cohen, S., and Istas, J. "Identification of the Hurst index of a Step Fractional Brownian Motion.", S.I.S.P., Vol. 3, Issue 1/2, p.101-111, 2000.

[6] Benassi, A., Jaffard, S. and Roux, D., "Elliptic Gaussian random processes", Rev. Mathemàtica Iberoamericana, vol. 13, No 1, p. 19-90, 1997.

[7] Bertrand, P."A local method for estimating change points : the hat-function ", Statistics 34, p.215-235, (2000). 
[8] Cheridito, P. "Arbitrage in fractional Brownian motion models", to appear in Finance and Stochastics.

[9] Davydov, Y., Khoshnevisan, D., Shi, Z., and Zitikis, R., "Convex rearrangements, generalized Lorenz curves, and correlated Gaussian data", preprint PMA-825, 2003.

[10] Guyon, X., and Leon, J.R., "Convergence en loi des H-variations d'un processus gaussien fractionnaire", Ann. Inst. H. Poincaré, 25, n³, p.265-282, 1989.

[11] Istas, J. and Lang, G., "Quadratic variation and estimation of the local Hölder index of a Gaussian process", Ann. Inst. H. Poincaré, 33 n4, pp.407-437, 1997.

[12] Kolmogorov, "Wienersche Spiralen und einige andere interessante Kurven in Hilbertchen Raume", Doklady, 26, p.115-118, 1940.

[13] Mandelbrot, B., and Van Ness, J. , Fractional Brownian motion, fractional noises and applications, SIAM review 10, p.422-437, 1968.

[14] Papanicolaou, G. and Sølna, K. Wavelet Based Estimation of Local Kolmogorov Turbulence, in Long-range Dependence: Theory and Applications, P. Doukhan, G. Oppenheim and M.S. Taqqu editors, Birkhäuser, 2002.

[15] Peltier, R., and Lévy Véhel, J., "Multifractional Brownian motion : definition and preliminary results", Rapport technique INRIA, 1996.

[16] Samorodnitsky, G. and Taqqu M. S. , Stable non-Gaussian Random Processes, Chapman \& Hall, 1994. 